\title{
STRESS-DILATANCY FOR SOILS. PART III: EXPERIMENTAL VALIDATION FOR THE BIAXIAL CONDITION
}

\author{
ZENON SZYPCIO \\ Department of Civil and Environmental Engineering, \\ Białystok University of Technology, Białystok, Poland, e-mail: z.szypcio@pb.edu.pl
}

\begin{abstract}
The validation of the general stress-dilatancy relationship is shown based on biaxial compression test data presented in the literature under drained and undrained conditions. Rowe's and Bolton's relationships can be treated as simplified forms of the general stress-dilatancy relationship. The stress ratio values are a function of not only the dilatancy but also the intermediate principal stress, the non-coaxiality angle defined by Gutierrez and Ishihara and the stress-strain path. For many granular soils, the critical frictional state angle of the shearing resistance $\Phi^{o}=\Phi_{c v}^{\prime}$ and parameters $\alpha$ and $\beta$ are functions of the drainage condition, the stress level and the stress and strain paths.
\end{abstract}

Key words: soil mechanics, stress-dilatancy, biaxial compression

\section{INTRODUCTION}

Most geotechnical structures such as slopes, embankments, retaining walls, and strip foundations can be simplified into plane strain conditions, which are modelled in a plane strain biaxial apparatus [2], [5], [6], [25], [26], [30] and others or in true triaxial tests [18].

Rowe's stress-dilatancy relation was one of the first rational attempts to characterize the dilatancy of soils in plane strain conditions [19], [20]. The modified non-coaxial version of Rowe's stress-dilatancy relationship was proposed by Gutierrez and Wang [10]. Intermediate stress $\left(\sigma_{2}^{\prime}\right)$ is known to play an important role in the stress-strain behaviour [14] but is not considered in the original Rowe [19] or modified Gutierrez and Wang [10] stress-dilatancy relations.

The stress, the strain, the stress and strain history and the stress level play important roles in the stressstrain behaviour of soil in shearing, especially in plane strain conditions ([18], [23], [25], [31], [32]).

The effects of the anisotropy and non-homogeneity on the stress-strain behaviour of soils cannot be neglected in soil modelling and laboratory tests. The anisotropy and non-homogeneity result from the formation history in the field and the sample preparation method in the laboratory ([16], [24], [27] and many others). The non-homogeneity is visible in many plane strain tests as the formation of a shear band. The persistent shear band forms at mobilized friction levels very close to the peak value ([7], [8], [11], [24], [26]).

As a consequence, the stress-strain (dilatancy) relationship is affected by many factors, and validating the theoretical stress-dilatancy relationship is very difficult.

This paper presents the validation of the general stress-dilatancy relationship developed by Szypcio [21] for plane strain conditions. Soil is treated as an isotropic continuum, and the rheology, breakage of grains, temperature changes and other non-mechanical effects are neglected. The influences of the intermediate stress $\left(\sigma_{2}^{\prime}\right)$ and non-coaxiality are analysed for drained and undrained conditions based on the frictional state theory [21] for experimental data presented in the literature. Only the pre-peak phase (stage), when the deformation is homogeneous, is analysed.

\section{STRESS-DILATANCY RELATIONSHIPS FOR PLANE STRAIN}

The general stress-plastic dilatancy relationship [21] has the form

$$
\eta=Q_{b}-A_{b} D^{p}
$$


where

$$
\begin{gathered}
Q_{b}=M_{b}^{o}-\alpha A_{b}^{o}, \\
A_{b}=\beta A_{b}^{o} .
\end{gathered}
$$

Subscript $b$ indicates the plane (biaxial) strain condition. The stress ratio is

$$
\eta=\frac{q}{p^{\prime}}
$$

where

$$
\begin{gathered}
p^{\prime}=\frac{1}{3}\left(\sigma_{1}^{\prime}+\sigma_{2}^{\prime}+\sigma_{3}^{\prime}\right), \\
q=\sqrt{\sigma_{1}^{\prime 2}+\sigma_{2}^{\prime 2}+\sigma_{3}^{\prime 2}-\sigma_{1}^{\prime} \sigma_{2}^{\prime}-\sigma_{1}^{\prime} \sigma_{3}^{\prime}-\sigma_{2}^{\prime} \sigma_{3}^{\prime}} .
\end{gathered}
$$

The frictional state characteristic values [21] are

$$
\begin{gathered}
M_{b}^{o}=\frac{3 \sin \Phi^{o}}{\sqrt{3} \cos \theta_{b}-\sin \Phi^{o} \sin \theta_{b}}, \\
A_{b}^{o}=\frac{1}{\cos \left(\theta_{b}-\theta_{\varepsilon}\right)}\left\{1-\frac{2}{3} M_{b}^{o} \sin \left(\theta_{b}+\frac{2}{3} \pi\right)\right\}
\end{gathered}
$$

for drained conditions,

$$
A_{b}^{o}=\frac{1}{\cos \left(\theta_{b}-\theta_{\varepsilon}\right)}\left\{1-\frac{2}{3} M_{b}^{o} \sin \left(\theta_{b}-\frac{2}{3} \pi\right)\right\}
$$

for undrained conditions,

$$
\theta_{b}=\tan ^{-1} \frac{\sigma_{1}^{\prime}+\sigma_{3}^{\prime}-2 \sigma_{2}^{\prime}}{\sqrt{3}\left(\sigma_{1}^{\prime}-\sigma_{3}^{\prime}\right)}
$$

where $\Phi^{o}$ is the angle of shear resistance at the critical frictional state.

The plastic dilatancy is

$$
D^{p}=\frac{\delta \varepsilon_{v}^{p}}{\delta \varepsilon_{q}^{p}}
$$

where

$$
\begin{aligned}
& \delta \varepsilon_{v}^{p}=\delta \varepsilon_{v}-\delta \varepsilon_{v}^{e}, \\
& \delta \varepsilon_{q}^{p}=\delta \varepsilon_{q}-\delta \varepsilon_{q}^{e} .
\end{aligned}
$$

For plane strain conditions, $\delta \varepsilon_{2}=0$

$$
\begin{gathered}
\delta \varepsilon_{v}=\delta \varepsilon_{1}+\delta \varepsilon_{3}, \\
\delta \varepsilon_{q}=\frac{2}{3} \sqrt{\delta \varepsilon_{1}^{2}+\delta \varepsilon_{3}^{2}-\delta \varepsilon_{1} \delta \varepsilon_{3}} .
\end{gathered}
$$

The elastic parts of the strain increment invariants are

$$
\begin{gathered}
\delta \varepsilon_{v}^{e}=\frac{\kappa}{1+e} \frac{\delta p^{\prime}}{p^{\prime}} \\
\delta \varepsilon_{q}^{e}=\frac{2}{9} \frac{\kappa(1+v)}{(1+e)(1-2 v)} \frac{\delta q}{p^{\prime}} .
\end{gathered}
$$

where $v$ is Poisson's ratio, $e$ is the void ratio, and $\kappa$ is the slope of the unloading/reloading line in the $e-\ln p^{\prime}$ plane.

Assuming "full plane" conditions, $\delta \varepsilon_{2}=0$ as well as $\delta \varepsilon_{2}^{p}=0$; therefore, we can write

$$
\begin{gathered}
\delta \varepsilon_{v}^{p}=\delta \varepsilon_{1}^{p}+\delta \varepsilon_{3}^{p}=\delta \varepsilon_{1}^{p}\left(1+D^{*}\right), \\
\delta \varepsilon_{q}^{p}=\frac{2}{3} \delta \varepsilon_{1}^{p} \sqrt{1-D^{*}+D^{* 2}}
\end{gathered}
$$

where

$$
D^{*}=\frac{\delta \varepsilon_{3}^{p}}{\delta \varepsilon_{1}^{p}}
$$

Plastic dilatancy may have the following form

$$
D^{p}=\frac{2}{3} \frac{1+D^{*}}{\sqrt{1-D^{*}+D^{* 2}}} .
$$

The principal strain increments can be calculated from the strain invariant increments [21]

$$
\begin{gathered}
\delta \varepsilon_{1}^{p}=\frac{1}{3} \delta \varepsilon_{v}^{p}-\delta \varepsilon_{q}^{p} \sin \left(\theta_{\varepsilon b}-\frac{2}{3} \pi\right), \\
\delta \varepsilon_{2}^{p}=\frac{1}{3} \delta \varepsilon_{v}^{p}-\delta \varepsilon_{q}^{p} \sin \theta_{\varepsilon b} \\
\delta \varepsilon_{3}^{p}=\frac{1}{3} \delta \varepsilon_{v}^{p}-\delta \varepsilon_{q}^{p} \sin \left(\theta_{\varepsilon b}+\frac{2}{3} \pi\right) .
\end{gathered}
$$

In this paper, it is assumed that $\delta \varepsilon_{1}^{p} \geq \delta \varepsilon_{2}^{p} \geq \delta \varepsilon_{3}^{p}$; therefore, $-\frac{\pi}{6} \leq \theta_{\varepsilon b} \leq \frac{\pi}{6}$.

For "full plane" conditions, $\delta \varepsilon_{2}^{p}=0$; therefore

$$
\sin \theta_{\varepsilon b}=\frac{1}{3} D^{p}=\frac{1}{2} \frac{1+D^{*}}{\sqrt{1-D^{*}+D^{* 2}}} .
$$

The relationship between $\theta_{\varepsilon b}$ and $D^{*}$ is shown in Fig. 1.

The angle $-\frac{\pi}{6} \leq \theta_{\varepsilon b} \leq \frac{\pi}{6}$ for $D^{*} \leq 0$. Similar to the strain increment, the principal stress can be calculated from the stress invariants [21] 


$$
\begin{gathered}
\sigma_{1}^{\prime}=p^{\prime}-\frac{1}{3} q \sin \left(\theta_{b}-\frac{2}{3} \pi\right), \\
\sigma_{2}^{\prime}=p^{\prime}-\frac{1}{3} q \sin \theta_{b}, \\
\sigma_{3}^{\prime}=p^{\prime}-\frac{1}{3} q \sin \left(\theta_{b}-\frac{2}{3} \pi\right) .
\end{gathered}
$$

Because $\sigma_{1}^{\prime} \geq \sigma_{2}^{\prime} \geq \sigma_{3}^{\prime}, \sigma_{1}^{\prime} / \sigma_{2}^{\prime} \geq 1$ and $\sigma_{1}^{\prime} / \sigma_{3}^{\prime} \geq 1$; this paper considers only these stress paths.

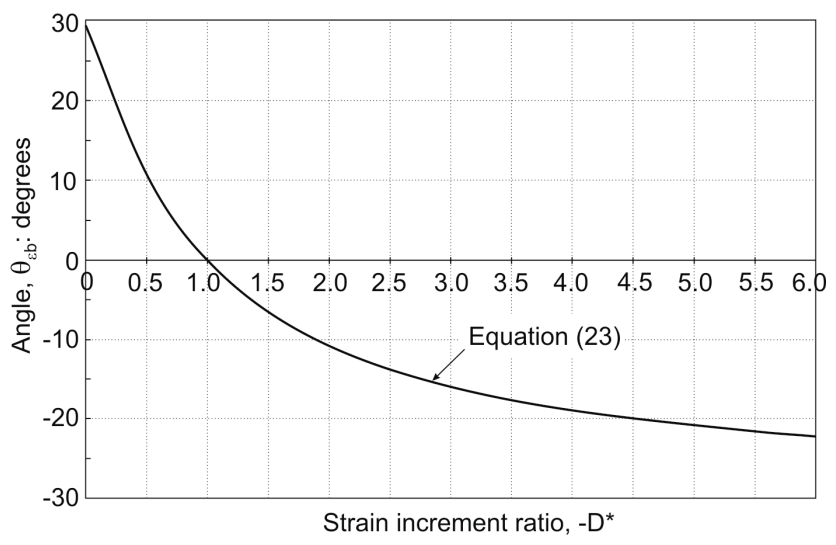

Fig. 1. Relationship between $\theta_{\varepsilon b}$ and $D^{*}$

Under biaxial compression, the value of angle $\theta_{b}$ quickly decreases from approximately $30^{\circ}$ to the minimum value during shearing and remains almost constant for the pre-failure stage ([5], [15], [27], [30]).

Tatsuoka et al. [23] showed that the value of $b$ at the peak is between 0.2 and $0.3\left(13^{\circ}<\theta_{b}<19^{\circ}\right)$. Pradhan et al. [17] observed $b$ values ranging between 0.22 and $0.33\left(11^{\circ}<\theta_{b}<18^{\circ}\right)$. The value of $b$ (angle $\theta_{b}$ ) depends on the contact of the belt platen, the initial porosity [9] and the height-to-width ratio of the sample [14].

The stress ratio can be written as

$$
\frac{\sigma_{1}^{\prime}}{\sigma_{3}^{\prime}}=\frac{1-\frac{2}{3} \eta \sin \left(\theta_{b}-\frac{2}{3} \pi\right)}{1-\frac{2}{3} \eta \sin \left(\theta_{b}+\frac{2}{3} \pi\right)}
$$

or

$$
\frac{\sigma_{1}^{\prime}}{\sigma_{3}^{\prime}}=\frac{1-\frac{2}{3} \sin \left(\theta_{b}-\frac{2}{3} \pi\right)\left\{M_{b}^{o}-A_{b}^{o}\left(\alpha+\beta D^{p}\right)\right\}}{1-\frac{2}{3} \sin \left(\theta_{b}+\frac{2}{3} \pi\right)\left\{M_{b}^{o}-A_{b}^{o}\left(\alpha+\beta D^{p}\right)\right\}} .
$$

Equation (26) is the general stress-strain equation for the plane strain (biaxial) condition.
For granular (non-cohesive) soils, the effective mobilized angle of friction can be calculated from the equation

$$
\Phi^{\prime}=\tan ^{-1}\left(\sqrt{\sigma_{1}^{\prime} / \sigma_{3}^{\prime}}\right)-\frac{\pi}{2} .
$$

Thus, the values of $\sigma_{1}^{\prime} / \sigma_{3}^{\prime}$ and $\Phi^{\prime}$ are functions of $\Phi^{o}, \theta_{b}, \theta_{\varepsilon b}, \alpha, \beta$ and $D^{*}$.

In the frictional state theory, it is assumed that the angle $\Phi^{o}$ is characteristic of soils and does not depend on the deformation mode [21]. For many noncohesive soils, it can be assumed that $\Phi^{o}=\Phi_{c v}^{\prime}$ [22].

The plastic work increment for "full plane" conditions is

$$
\delta W^{*}=\sigma_{1}^{\prime} \delta \varepsilon_{1}^{p}+\sigma_{3}^{\prime} \delta \varepsilon_{3}^{p} \geq 0 .
$$

This paper considers only the deformation process for which

$$
\frac{\sigma_{1}^{\prime}}{\sigma_{3}^{\prime}} \geq-D^{*}
$$

The influence of $\theta_{b}$ on the stress ratio $\left(\sigma_{1}^{\prime} / \sigma_{3}^{\prime}\right)$ for biaxial compression is shown in Fig. 2.

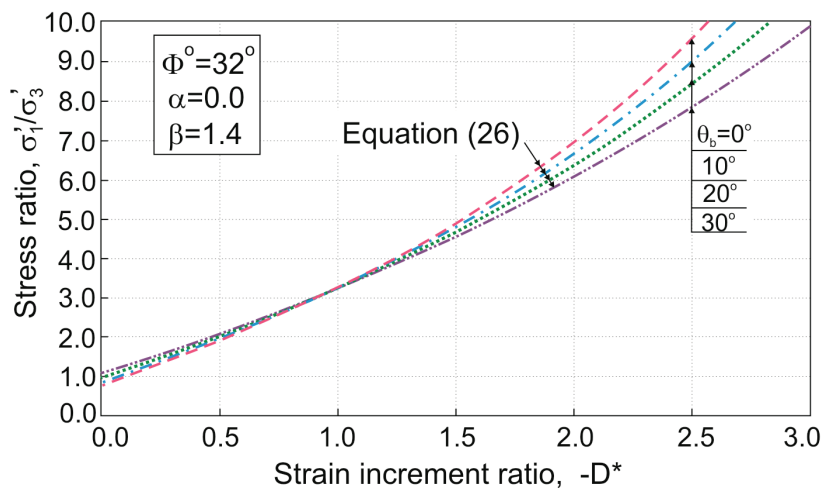

Fig. 2. Influence of angle $\theta_{b}$ on the stress ratio

Angle $\theta_{b}$ (intermediate stress $\sigma_{2}^{\prime}$ ) influences the stress ratio; this may be due to the assumption of "full plane" conditions in this paper, but further investigation is needed.

\section{COMPARISON WITH ROWE AND BOLTON THEORIES}

The Rowe's stress-strain relationship ([19], [20]) for drained plane strain conditions can be expressed by the equation 


$$
\frac{\sigma_{1}^{\prime}}{\sigma_{3}^{\prime}}=-K \frac{\delta \varepsilon_{3}}{\delta \varepsilon_{1}}
$$

where the experimental constant $K=\tan ^{2}\left(45^{\circ}+\right.$ $\left.\Phi_{c v}^{\prime} / 2\right)$.

Bolton [3] showed that Rowe's stress-strain relationship can be expressed in the form

$$
\Phi^{\prime}=\Phi_{c v}^{\prime}+0.8 \psi
$$

where the dilatancy angle can be calculated from the following equation

$$
\sin \psi=-\frac{\delta \varepsilon_{1}+\delta \varepsilon_{3}}{\delta \varepsilon_{1}-\delta \varepsilon_{3}} .
$$

For the drained condition, the elasticity part of the strain increment can be treated as small, and equations (30) and (32) have the forms

$$
\begin{gathered}
\frac{\sigma_{1}^{\prime}}{\sigma_{3}^{\prime}}=-K D, \\
\sin \psi=-\frac{1+D}{1-D},
\end{gathered}
$$

where $D=D^{*}=\delta \varepsilon_{3} / \delta \varepsilon_{1}$.

Rowe's and Bolton's relationships between the stress ratio and dilatancy are independent of the intermediate stress $\theta_{b}$. Therefore, for comparison, it is assumed that $\theta_{b}=15^{\circ}$ as the mean value observed in drained biaxial compression tests.

Figures 3 and 4 show that the best approximation of Rowe's and Bolton's equations (equations (30) and (31), respectively) are obtained for $\Phi^{o}=\Phi_{c v}^{\prime}, \alpha=0$ and $\beta=1.4$.

Very good conformity is obtained for $\sigma_{1}^{\prime} / \sigma_{3}^{\prime}>K$ and $\sigma_{1}^{\prime} / \sigma_{3}^{\prime}<2 K(-2 \leq D \leq-1)$, which are treated as maximum values for Rowe's theory ([20], [12], [13]). Poorer conformity is obtained for $-1<D \leq 0$.

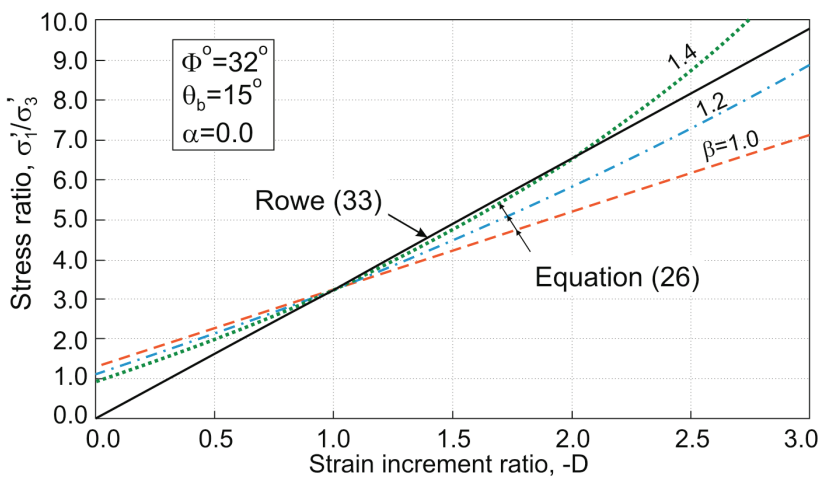

Fig. 3. Comparison of Rowe's and frictional state theory relationships between the stress ratio and the strain increment ratio

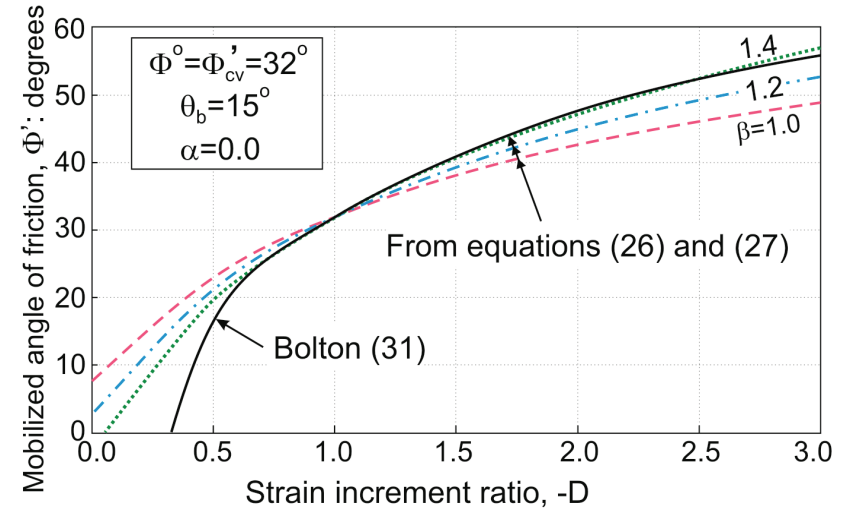

Fig. 4. Comparison of Bolton's and the frictional state theory relationships

Very good conformity is obtained with Bolton's and the frictional state theory relationships for $-3<D^{*}$ $<-0.5$ (Fig. 4). Contrary to the triaxial condition, $\beta \neq 1$ for granular soil in the plane strain condition [21]. This difference is perhaps due to a smaller degree of freedom for interparticle movement under plane strain than under triaxial conditions [1]. Thus, the mode of deformation influences the parameters of the frictional state theory.

Bolton [3], analysing the relationship between the strength and dilatancy of quartz sands in plane strain conditions at different densities and confirming pressures, found the following correlations

$$
\begin{gathered}
\Phi_{\max }^{\prime}-\Phi_{c v}^{\prime}=5 I_{R}, \\
\left(-\frac{\delta \varepsilon_{v}}{\delta \varepsilon_{1}}\right)=0.3 I_{R} .
\end{gathered}
$$

The relative density index for quartz sands is

$$
I_{R}=I_{D}\left(10-\ln p^{\prime}\right)-1
$$

where $p^{\prime}$ is expressed in kilonewtons per square metre $(\mathrm{kPa})$ and $I_{D}$ is the density index.

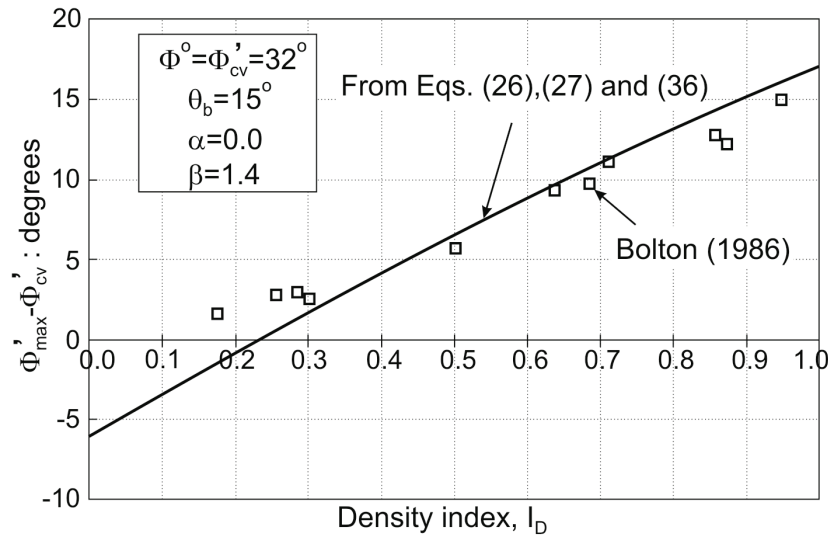

Fig. 5. Influence of density index on $\left(\Phi_{\max }^{\prime}-\Phi_{c v}^{\prime}\right)$ 
Figure 5 shows the values of $\Phi_{\max }^{\prime}-\Phi_{c v}^{\prime}$ collected by Bolton [3] and calculated from equations (26) and (27) for $\Phi^{o}=\Phi_{c v}^{\prime}=32^{\circ}, \theta_{b}=15^{\circ}, \alpha=0$, and $\beta=1.4$.

Bolton's equation, given by equation (35), can be treated as an approximation of the stress-dilatancy equation given in (1) obtained from the frictional state theory.

\section{STRESS-DILATANCY FOR DRAINED CONDITIONS}

Brasted sand was investigated in plane strain conditions by Cornforth [5]. The values of the maximum angle of friction $\Phi_{\max }^{\prime}$ for different initial porosities obtained experimentally and calculated from equations (26) and (27) are shown in Fig. 6. At failure, $\delta p^{\prime}=0, \delta \varepsilon_{v}^{e}=0$ and $D^{p}=D$.

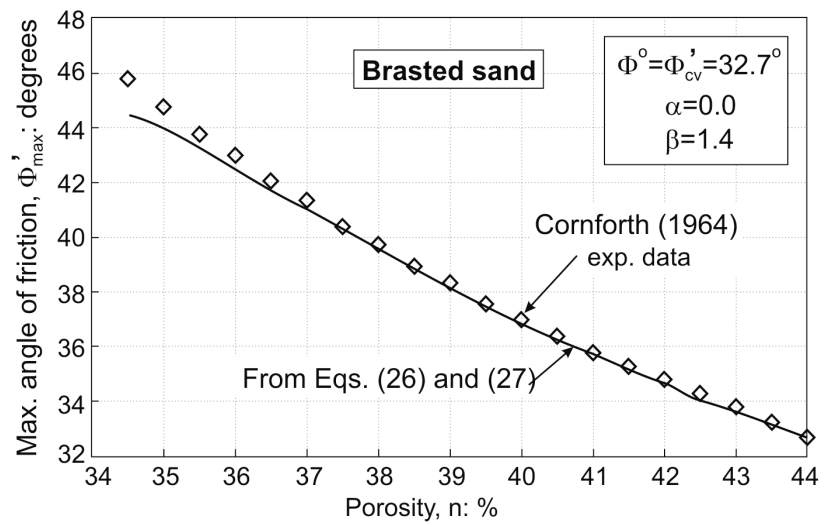

Fig. 6. Maximum friction angle of Brasted sand

The calculations used the value of $\theta_{b}$ obtained in the experiment [5], $\Phi^{o}=\Phi_{c v}^{\prime}=32.7^{\circ}, \alpha=0$ and $\beta=1.4$. The frictional state theory clearly approximates the experimental data very well.

Changi sand was extensively investigated by Wanatowski ([28], [29], [30]) and Wanatowski and Chu [27] in plane strain conditions. Figure 7 shows the relationship between the stress ratio $(\eta)$ and plastic dilatancy $\left(D^{p}\right)$ for three tests [30]. The samples were anisotropically consolidated and sheared under drained conditions at a constant $\sigma_{3}^{\prime}$.

The $\eta-D^{p}$ relationship is approximately linear throughout the pre-peak stage. The calculations were made with $\kappa=0.008, v=0.30$ and $\Phi^{o}=\Phi_{c v}^{\prime}=33.4^{\circ}$. The best approximation of the experimental data was obtained assuming $\alpha=-0.29$ and $\beta=1.2$ for
CKoD01, $\alpha=-0.30$ and $\beta=0.80$ for CKoD02 and $\alpha=-0.32$ and $\beta=0.90$ for CKoD03 tests. Before failure, the parameters $\alpha$ and $\beta$ depend on the consolidation pressure and other unidentified factors. Parameter $\alpha$ represents the translation, while $\beta$ represents the slope of the $\eta-D^{p}$ line. The translation and different slopes of the $\eta-D^{p}$ lines were also observed by Yasin and Tatsuoka [31] for Toyoura sand tested in plane strain conditions for different stress paths.

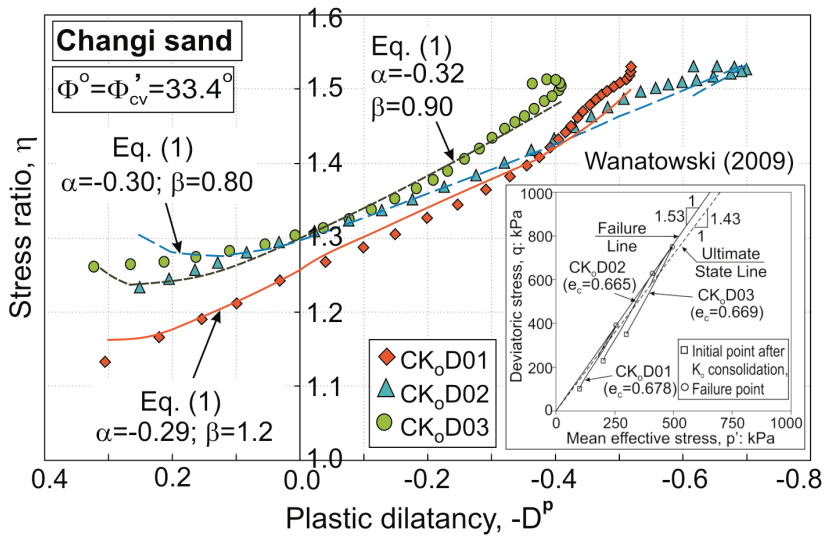

Fig. 7. Relationship between the stress ratio and plastic dilatancy for Changi sand

Masuda et al. [15] extensively investigated the stress-strain behaviour of Toyoura sand in plane strain condition under monotonic and cyclic loading and unloading. The experiments were executed in a modified plane strain compression apparatus on specially prepared samples consolidated both isotropically and anisotropically. An analysis of the experimental data of these tests shows that a mean value of $\theta_{b} \approx 22.5$. The relationship between the stress ratio $\left(\sigma_{1}^{\prime} / \sigma_{3}^{\prime}\right)$ and the plastic strain increment ratio $\left(D^{*}\right)$ for loading and unloading are shown in Figs. 8 and 9, respectively.

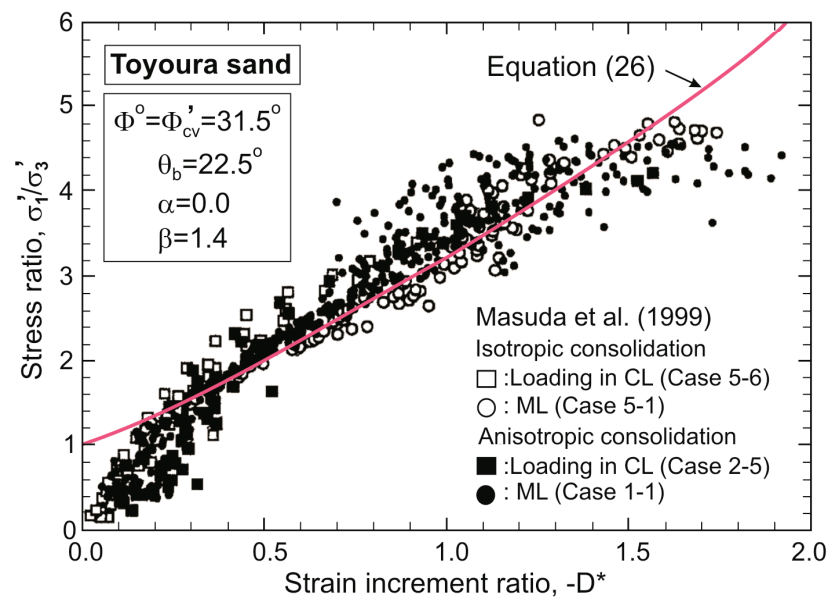

Fig. 8. Relationship between the stress ratio and the strain increment ratio for biaxial loading tests on Toyoura sand 


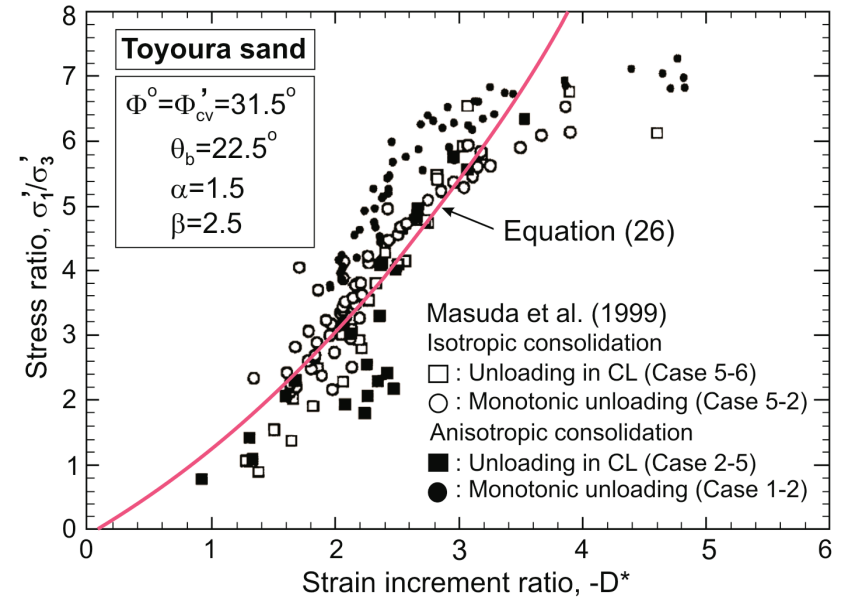

Fig. 9. Relationship between the stress ratio and the strain increment ratio for biaxial unloading tests on Toyoura sand

An approximation of the experimental data was obtained from the frictional state theory (equation (26)) for $\Phi^{o}=\Phi_{c v}^{\prime}=31.5^{\circ}$ and $\theta_{b}=22.5$, with $\alpha=0$ and $\beta=1.4$ for loading and $\alpha=1.5$ and $\beta=2.5$ for unloading. The stress-strain behaviour of Toyoura sand is clearly very different in the loading and unloading tests.

\section{STRESS-PLASTIC DILATANCY FOR UNDRAINED CONDITIONS}

For undrained conditions, $\delta \varepsilon_{v}=0$; therefore, $\delta \varepsilon_{v}^{p}=$ $-\delta \varepsilon_{v}^{e}=-\frac{\kappa}{1+e} \frac{\delta p^{\prime}}{p^{\prime}}$.

The validation of the stress-plastic dilatancy relationship obtained from the frictional state theory (equation (1)) is conducted based on the original experimental data of three tests on medium dense and two tests on very loose Changi sand [30].

The medium dense sand was first $K_{o}$ consolidated to varying mean effective stresses and sheared under undrained conditions in the deformation-controlled loading mode. Strain-hardening behaviour was observed in the tests. The effective stress path asymptotically approaches a straight line that is called the critical state ratio line (CSRL, [4]) with a gradient of $M_{L}=1.39$. The stress ratio-plastic dilatancy relationships for the three tests (CKoU01, CKoU02, and CKoU03) are shown in Fig. 10. The calculations were made with $\kappa=0.025$ and $v=0.30$. For all three tests, the $\eta-D^{p}$ relationships are very similar and well approximated by the stress ratio-plastic dilatancy relationship obtained from the frictional state theory for $\Phi^{o}=\Phi_{c v}^{\prime}=33.4^{\circ}, \alpha=-0.175$ and $\beta=$ -0.09 (Fig. 10).

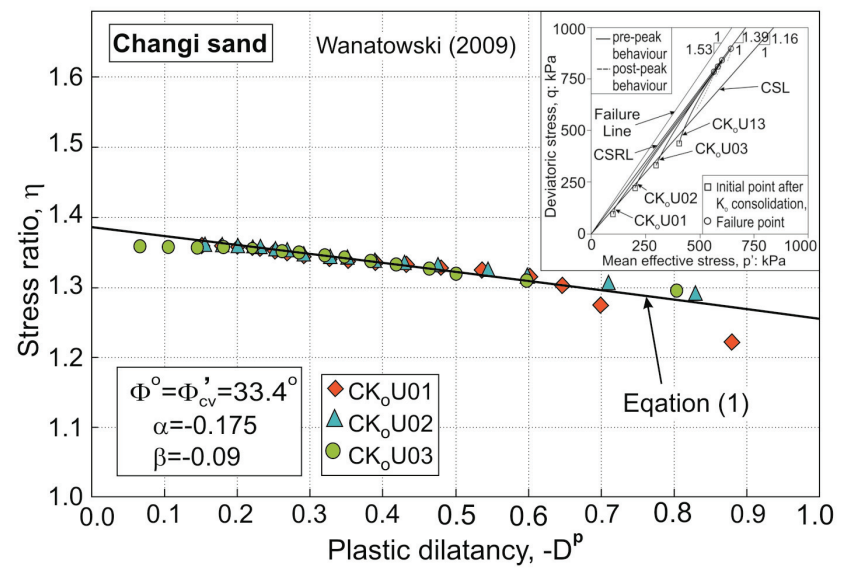

Fig. 10. Stress ratio-plastic dilatancy relationship for the strain-hardening behaviour of Changi sand

The values of parameters $\alpha$ and $\beta$ are characteristically negative.

Some tests on very loose Changi sand were conducted in undrained conditions. The specimens were $K_{o}$ consolidated to varying mean effective stresses and sheared under a load-controlled loading mode. When the peak deviatoric stress was reached, the axial strain suddenly increased, and the specimen collapsed; however, a shear band was not observed in these tests [30]. The stress ratio-plastic dilatancy relationships for two of the tests (CKoU05 and CKoU06) are shown in Fig. 11. For these two tests, the $\eta-D^{p}$ relationships calculated for $\kappa=0.008$ and $v=0.30$ are very similar and well approximated by equation (1) for $\Phi^{o}=\Phi_{c v}^{\prime}=33.4^{\circ}, \alpha=-0.11$ and $\beta=1.10$. Similar to the case of medium dense sand, parameter $\alpha$ is negative, while parameter $\beta$ is positive.

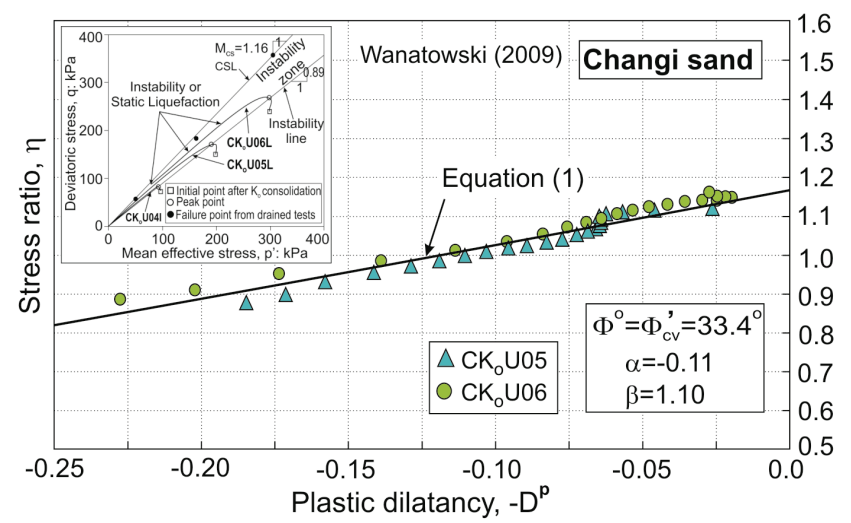

Fig. 11. Stress ratio-plastic dilatancy relationship for the collapse behaviour of Changi sand 
The frictional state theory describes well the stress-strain relationship in undrained plane strain conditions.

\section{CONCLUSIONS}

The stress-dilatancy relationship obtained from the frictional state theory can describe the real behaviour of non-cohesive soils in plane strain conditions.

Rowe's and Bolton's equations can be treated as approximations of the stress-dilatancy relationship presented in this paper.

The mean stress (angle $\theta_{b}$ ) and non-coaxiality angle $\left(\theta_{b}-\theta_{\varepsilon b}\right)$ influence the stress-strain relationship in plane strain conditions.

Based on the experimental data investigated for many different sands, $\Phi^{o}=\Phi_{c v}^{\prime}$. The values of parameters $\alpha$ and $\beta$ can be assumed to be constant during shearing and are functions of the drainage conditions, the stress level and the stress and strain paths.

The complete validation of the stress-dilatancy relationship in plane strain conditions requires further experimental investigation, especially for cohesive soils for different stress-strain paths.

\section{ACKNOWLEDGEMENTS}

The author would like to thank Prof. D. Wanatowski for the original experimental data on the plane strain compression of Changi sand.

\section{REFERENCES}

[1] Barden L., Khayatt A.J., Wightman A., Elastic and slip components of the deformation of sand, Canadian Geotechnical J., 1969, Vol. 6, No. 3, 227-240.

[2] Bishop A.W., Test requirements of measuring the coefficient of earth pressure at rest, Proc. European Conf. Earth Pressure Problems, Brussels, 1958, 1, 2-14.

[3] Bolton M.D., The strength and dilatancy of sands, Geotechnique, 1986, 36, No. 1, 65-78.

[4] Chu J., LeroueIL S., LeONG W.K., Unstable behaviour of sand and its implication for slope stability, Canadian Geotechnical J., 2003, Vol. 40, No. 5, 873-885.

[5] CORNFORTH D.H., Some experiments on the influence of strain conditions on the strength of sand, Geotechnique, 1964, 14, No. 2, 143-167.

[6] Drescher A., Vardoulakis I., Han C., A biaxial apparatus for testing soils, Geotech. Testing J. ASTM, 1990, 13, No. 3, 226-234.
[7] Finno R.J., Alarcon M.A., Mooney M.A., Viggiani G., Shear bands in plane strain active tests of moist tamped and pluviated sands, Proc. of the Fount. Int. Conf. on Soil Mech. and Found. Eng., Hamburg, 1997, 295 $-298$.

[8] Gajo A., BigOni D., Wood M., Multiple shear band development and related instabilities in granular materials, J. Mech. Phus. Solids, 2004, 52, No. 12, 2683-2724.

[9] GReEn G.E., ReAdES D.W., Boundary conditions, anisotropy and sample shape effects on the stress-strain behaviour of sand in triaxial compression and plane strain, Geotechnique, 1975, 25, No. 2, 333-356.

[10] Gutierrez M., WANG J., Non-coaxial version of Rowe's stress-dilatancy relation, Granular Matter, 2009, Vol. 11, 129-137.

[11] Han C., Drescher A., Shear bands in biaxial tests on dry coarse sand, Soils and Foundations, 1993, Vol. 33, No. 1, 118-132.

[12] Horne M.R., The Behaviour of an Assembly of Rotund, Rigid, Cohesionless Particles. I. Proceedings of the Royal Society of London. Series A. Mathematical and Physical Sciences, 1965, Vol. 286, No. 1404, 62-78.

[13] Horne M.R., The Behaviour of an Assembly of Rotund, Rigid, Cohesionless Particles. II. Proceedings of the Royal Society of London. Series A. Mathematical and Physical Sciences, 1965, Vol. 286, No. 1404, 79-97.

[14] LAM W.-K., TATSUOKA F., Effects of initial anisotropic fabric and $\sigma_{2}$ on strength and deformation characteristics of sand, Soils and Foundations, 1988, Vol. 28, No. 1, 89-106.

[15] Masuda T., Tatsuoka F., Yamada S., Sato T., Stressstrain behaviour of sand in plane strain compression, extension and cyclic loading tests, Soils and Foundations, 1999, Vol. 39, No. 5, 31-45.

[16] Oda M., KoISHIKawa I., Higuchi T., Experimental study of anisotropic shear strength of sand by plane strain test, Soils and Foundations, 1978, Vol. 18, No. 1, 25-38.

[17] Pradhan T.B.S., Tatsuoka F., HoriI N., Strength and deformation characteristics of sand in torsional simple shear, Soils and Foundations, 1988, Vol. 28, No. 3, 131-148.

[18] Roscoe K.H., The influence of strains in soil mechanics, Geotechnique, 1970, 25, No. 2, 333-356.

[19] Rowe P.W., The stress-dilatancy relation for static equilibrium of an assembly of particles in contact, Proc. Roy. Soc. Sen. A., 1962, 269, 500-527.

[20] Rowe P.W., The relation between the shear strength of sands in triaxial compression, plane strain and direct shear, Geotechnique, 1969, 36, No. 1, 65-78.

[21] Szypcio Z., Stress-dilatancy for soils. Part I: The frictional state theory, Studia Geotechnica et Mechanica, 2016, Vol. 38, No. 4, 51-57.

[22] Szypcio Z., Stress-dilatancy for soils. Part II: Experimental validation for triaxial tests. Studia Geotechnica et Mechanica, 2916, Vol. 38, No. 4, 59-65.

[23] TATSUOKa F., SAKamoto M., Kawamura T., FuKushima S., Strength and deformation characteristics of sand in plane strain compression at extremely low pressures, Soils and Foundations, 1986, Vol. 26, No. 1, 65-84.

[24] Tatsuoka F., Nakamura S., Huang C., Tani K., Strength anisotropy and shear bend direction in plane strain tests of sound, Soil and Foundations, 1990, Vol. 30, No. 1, 35-54. 
[25] TOPOLNICKI M., Observed stress-strain behaviour of remoulded saturated clay and examination of two constitutive models, Publ. of the Institute of Soil and Rock Mechanics in Karlsruhe, 1987, 107.

[26] VARDOULAKIS I., Shear bend inclination and shear modulus of sand in biaxial test, Int. J. Num. Anal. Meth. Geomech., 1980, 4, 103-119.

[27] Wanatowski D., Chu J., Effect of Specimen Preparation Method on the Stress-Strain Behaviour of Sand in PlaneStrain Compression Tests, Geotechnical Testing Journal, 2008, Vol. 31, No. 4, 308-320.

[28] Wanatowski D., Asymptotic behaviour of sand in planestrain compression tests, Studia Geotechnica et Mechanica, 2007, Vol. 29, No. 3-4, 1-26.
[29] WANATOWSKI D., Undrained instability of loose sand under plane-strain conditions and its engineering application, Foundations of Civil and Environmental Engineering, 2007, No. 10, 131-141.

[30] WanAtowski D., Strain softening and instability of sand. Experimental study under plane-strain conditions, VDM Verlag 2009.

[31] YASIN S.J.M., TATSUOKA F., Stress history-dependent deformation characteristics of dense sand in plane strain, Soils and Foundations, 2000, 40, No. 2, 77-98.

[32] Zhuang L., Nakata Y., Kim U.G., Kim D., Influence of relative density, particle shape, and stress path on the plane strain compression behaviour of granular materials, Acta Geotechnica, 2014, No. 9(2), 241-255. 